\title{
Delivering a Projectile Component to the Vestan Regolith
}

$2 \quad$ R. Terik Daly ${ }^{\mathrm{a}}$ and Peter H. Schultz ${ }^{\mathrm{a}}$

$3{ }^{\mathrm{a}}$ Department of Earth, Environmental and Planetary Sciences, Brown University, 324 Brook St.,

$4 \quad$ Box 1846, Providence, RI 02912

5 ronald_daly@brown.edu; peter_schultz@brown.edu

6 Corresponding author: R. Terik Daly (terik_daly@brown.edu; 408-455-4447)

\section{Abstract}

9 Dark material on Vesta may consist of carbonaceous chondrite-like material delivered by impact events. This study uses hypervelocity impact experiments to assess the viability of the

11 impact delivery hypothesis. Experiments reveal that impact events deliver significant fractions of

12 the projectile to the target during impacts at average Vestan speeds. Hence, dark material can

13 plausibly be delivered to Vesta by impacts, with the projectile component accumulating in the

14 regolith with time. Projectile retention is sensitive to impact angle, ranging from $7 \%$ for

$1530^{\circ}$ impacts (measured from horizontal) to $72 \%$ for vertical impacts. Averaged over the

16 probability distribution of impact angles, $17 \%$ of the projectile's mass remains in or near the

17 crater. Projectile-contaminated breccias largely remain inside the crater for vertical impacts. In

18 oblique impacts, projectile-contaminated pieces concentrate downrange beyond the crater rim.

19 Based on experiments, projectile delivery is expected for typical Vestan impact conditions, not

20 only for extraordinary events such as low-probability, very low-speed (e.g., $<2 \mathrm{~km} \mathrm{~s}^{-1}$ ) impacts.

21 These experiments indicate that other (non-dark) impactors contaminate the Vestan regolith.

22 Regolith-laden bodies in environments with similar impact speeds also may accrete significant 23 amounts of foreign debris. 
24 Keywords: Asteroid Vesta; asteroids, surfaces; impact processes; regoliths

\section{Introduction}

Impact processes sculpt the surfaces of the moons, planets, and small bodies in the Solar System. Some of these impacts leave lingering compositional traces of the projectile on the target object. The delivery of a projectile component is sensitive to many factors, including impact angle, impact speed, projectile composition, the presence of an atmosphere, and the material behavior of the projectile and target.

Asteroid 4 Vesta may be a particularly striking case of impactor contamination. Instruments on the Dawn spacecraft observed hydrated, low-albedo material, which many authors have interpreted to be remnants of carbonaceous chondrite-like impactors that collided with Vesta (Prettyman et al., 2012; McCord et al., 2012; Reddy et al., 2012; de Sanctis et al., 2012; Palomba et al., 2014; Jaumann et al., 2014; Turrini et al., 2014). Although impact delivery is these authors' favored explanation for dark material, the efficiency of the delivery process and physical state of the retained projectile component are poorly known. If projectile retention efficiency can be accurately determined, then the amount of dark material on Vesta may constrain the populations of dark and non-dark impactors and the degree of dynamical depletion in the main belt. The experiments reported here directly constrain the projectile retention efficiency of stony impactors for Vesta-like conditions.

Earlier studies by Pierazzo and Chyba (2002), Bland et al. (2008), Ong et al. (2010), Svetsov (2011), Bruck Syal and Schultz (2015), and Bruck Syal et al. (2015) have explored projectile delivery using numerical impact simulations. These studies, however, did not assess impactor delivery to Vesta specifically. In many cases, the types of impactors or impact speeds 
47 were not relevant to conditions at Vesta. The most relevant work was done by Turrini and

48 Svetsov (2014), who ran numerical impact models to calculate the water delivered to Vesta by

49 impacts of icy planetesimals. However, the retention efficiencies calculated in that study were

50 strongly influenced by the material model assumed in the simulations. It is unclear how

51 dramatically the projectile retention efficiencies calculated by Turrini and Svetsov (2014) are

52 affected by factors not included the simulations (e.g., shear). Current constitutive models and

53 equations of state do not accurately represent material behavior, which limits the ability of

54 impact models to represent projectile failure (Schultz and Stickle, 2011). Projectile failure,

55 however, affects the character of the delivered projectile component. Such problems are

56 especially acute for impacts at speeds less than $10 \mathrm{~km} \mathrm{~s}^{-1}$. Yet, more than $95 \%$ of impacts at

57 Vesta are slower than $10 \mathrm{~km} \mathrm{~s}^{-1}$ (O'Brien and Sykes, 2011). Numerical impact models also

58 cannot resolve mixing between the target and projectile, except for identifying cells occupied by

59 multiple materials (e.g., Artemieva and Pierazzo, 2011). However, mixing between target and

60 projectile may be very important for determining how the delivered projectile component is

61 expressed on a planetary surface.

62 In order to assess the viability of impact delivery as a source for Vestan dark material,

63 projectile retention needs to be assessed for stony projectiles impacting asteroidal regolith

64 analogs at impact speeds typical of Vesta. Impact experiments are ideal for doing this.

65 Experiments can be done at speeds typical of impacts at Vesta, using projectiles that are

66 excellent analogs for the Vestan impactors. Impact experiments can also use porous silicate

67 targets as an analog for the Vestan regolith. The use of porous targets is critical because target

68 porosity enhances projectile survival (Daly and Schultz, 2013; Bruck Syal and Schultz, 2015).

69 Experiments can also directly study detailed mixing relationships between target and projectile. 
Experiments assess the fraction of the impactor that is retained in and near the crater, as well as

71 the physical state (solid vs. particulate) of the delivered impactor fraction. Thus, experiments are

72 well suited to address how much of the impactor is delivered to and retained in the Vestan

73 regolith.

74

75 2. Materials \& Methods

76

2.1. Experiments at the NASA Ames Vertical Gun Range.

77

Experiments at the NASA Ames Vertical Gun Range (AVGR) assessed the retention of projectiles in proxy asteroidal regoliths (see Table 1). The AVGR is a two-stage light gas gun equipped with a large impact chamber ( 2.5 meters diameter, 2.5 meters tall) that readily

80 accommodates a variety of targets (Gault and Wedekind, 1978). Targets of porous, powdered

81 pumice ( $43 \%$ porosity; $\rho_{\text {target }} \approx 1.3 \mathrm{~g} \mathrm{~cm}^{-3}$ ) (Schultz et al., 2007) provided an analog for

82 asteroidal regoliths. Firing $1 / 4 "$ ' diameter basalt spheres at $30^{\circ}, 45^{\circ}, 60^{\circ}$, and $90^{\circ}$ (with respect to

83 horizontal) revealed the effect of impact angle on projectile retention. A $90^{\circ}$ experiment with a

84 1/4" diameter 2017-T4 aluminum sphere served as reference case for comparing brittle and ductile

85 projectiles. Impact speeds ranged from 4.5 to $5 \mathrm{~km} \mathrm{~s}^{-1}$, which are typical of collisions among

86 main belt asteroids and at Vesta (O’Brien and Sykes, 2011). After each experiment, breccias

87 contaminated by the projectile were recovered inside the crater and beyond the crater rim.

88

\subsection{Sample analysis.}

2.2.1. Micro-scale characterization.

Optical and scanning electron microscopy revealed the three-dimensional structure of

92 recovered projectile-contaminated breccias. A LEO 1530 variable pressure scanning electron 
93 microscope (SEM) equipped with a Robinson backscatter detector and Oxford instruments

94 electron dispersive spectroscopy (EDS) system was used to investigate chemical variations and

95 mixing relationships in pieces of projectile-contaminated breccias. Chemical data collected by

96 EDS were purely qualitative because the sample surface was neither polished nor flat. However,

97 these analyses required no sample preparation, which enabled studying chemical mixing between

98 projectile and target in full three-dimensional context.

99 Representative pieces of projectile-contaminated breccias were impregnated with low-

100 viscosity epoxy and thin sectioned for electron microprobe analysis. Thin sections were carbon

101 coated and analyzed using the Cameca SX 100 electron microprobe at Brown University (15 kV,

$10210 \mathrm{nA}$ current, $10 \mu \mathrm{m}$ beam diameter). All quantitative analyses were done with wavelength

103 dispersive spectroscopy. A loss routine (Devine et al., 1995) corrected for any sodium volatilized 104 during analysis. Microprobe analyses assessed projectile-target mixing in the transition from the 105 melt layer into the compressed zone of projectile-contaminated pieces (see section 3.4).

\section{2.2.2. Bulk Composition.}

108 Inductively-coupled plasma atomic emission spectroscopy (ICP-AES) characterized the 109 bulk chemical compositions of the pumice target, basalt projectiles, and projectile-contaminated 110 pieces that were not thin sectioned. ICP-AES samples were ground in an agate mortar and pestle 111 to $<200 \mu \mathrm{m}$ and then prepared using flux fusion and nitric acid digestion (Murray et al., 2000).

112 The resulting solutions were analyzed on a JY2000 Ultratrace ICP atomic emission spectrometer 113 at Brown University. 


\section{Results}

Individual pieces of projectile-contaminated breccias have two distinct sides, with an impact-melt breccia adhered to a shock-compressed zone of target pumice. On the impact-melt breccia side, vesicular glass encases melted blebs and occasional solid fragments of the projectile. Vesicles are pervasive in impact glasses from all experiments. This glassy zone reveals where the projectile and target mixed during the cratering process. On the reverse side of projectile-contaminated pieces, shock-compressed target pumice adheres to the melt (see Figure 1). The glassy impact melt breccia, not the shock-compressed pumice, bears the projectile component.

\subsection{Mass of the projectile retained.}

The mass of projectile-contaminated breccias recovered from an experiment does not directly reveal how much of the projectile was retained by the target. However, using a twocomponent chemical mixing model allows converting the mass of projectile-contaminated pieces into the projectile mass that was retained. To do so, the bulk compositions of the basalt projectiles, pumice targets, and projectile-contaminated breccias (see Tables 2 and 3) were reduced using the approach of Allegre et al. (1995). After normalizing analytical totals to 100\%, the concentration of each oxide was normalized by its standard deviation, multiplied by the concentration range, divided by the analytical error, and, finally, divided by the mean oxide concentration. This procedure accounts for analytical error and the spread in oxide abundances among the projectile, target, and projectile-contaminated pieces. Thus, oxides with small analytical errors and large concentration ranges are the most influential in the model. 
Following Cantagrel et al. (1984), two-component mixing is expressed as

$$
\left(C_{\text {Bas }}-C_{P u m}\right) X_{B a s}=\left(C_{P C P}-C_{P u m}\right)
$$

137 where $C_{B a s}$ and $C_{P u m}$ are the concentration of an oxide in the basalt projectile and pumice target, 138 respectively, $C_{P C P}$ stands for the concentration of an oxide in projectile-contaminated breccia 139 pieces, and $X_{B a s}$ is the mass fraction of the projectile in the projectile-contaminated pieces. For 140 each oxide, $\left(C_{P C P}-C_{P u m}\right)$ and $\left(C_{B a s}-C_{P u m}\right)$ is calculated from the reduced data and plotted 141 (Figure 2). Because this is a two-component mixing problem, if projectile-contaminated pieces 142 are a mixture of the basalt projectile and pumice target, then the oxide abundances in projectile143 contaminated pieces will plot on a line that passes through the origin. The slope of the line is $144 X_{\text {Bas }}$, which was calculated using least squares regression. The reduced data are highly linear for 145 all samples analyzed in this study (Figure 2). High coefficients of determination $\left(r^{2}>0.98\right.$, see 146 Table 4) indicate that essentially all variation is due to the linear relationship between $\left(C_{P C P}-\right.$ $\left.147 C_{\text {Pum }}\right)$ and $\left(C_{\text {Bas }}-C_{\text {Pum }}\right)$. These high values confirm that there is chemical negligible 148 contamination in the breccias due to a third endmember and increase our confidence in the 149 calculated $X_{B a s}$ values. The mass fraction of the projectile retained in and near the crater is 150 calculated by multiplying $X_{B a s}$ by the total mass of projectile-contaminated pieces recovered 151 from an experiment, divided by the original mass of the projectile (Table 4).

152 At vertical incidence, $72 \mathrm{wt} . \%$ of the projectile is retained in and near the crater (i.e., 153 within a few crater radii). Projectile retention efficiency decreases for oblique impacts, with only 1547 wt. \% of the projectile retained in and near the crater for a $30^{\circ}$ impact (Table 4). Empirically, 155 the data are matched by a $2^{\text {nd }}$ order polynomial $\left(r^{2}=0.9993\right)$ that assumes no projectile is 156 retained in or near the crater for a horizontal impact (Figure 3). We combined this empirical 157 projectile retention equation with the probability distribution of impact angles (Gilbert, 1893) to 
create a Monte Carlo model that calculated an angle-averaged projectile retention. The model indicates an average local projectile retention efficiency of $17 \mathrm{wt} . \%$ for basalt projectiles impacting asteroidal regolith analogs at 4.5 to $5 \mathrm{~km} \mathrm{~s}^{-1}$.

\subsection{Mass-frequency distribution of projectile-contaminated pieces.}

The cumulative mass-frequency distribution (MFD) of projectile-contaminated pieces affords insight into how the projectile component is distributed in and around the crater (Figure 4). In these figures we have normalized the individual and cumulative masses of projectilecontaminated pieces to the projectile mass to facilitate inter-experiment comparisons. Several massive pieces dominate the distributions, particularly for the $30^{\circ}$ and $45^{\circ}$ experiments, as indicated by the steep slope in the distributions above $M_{i} / M_{p} \sim 0.1$. All distributions plateau at small masses. The mass of projectile-contaminated pieces recovered from each experiment decreased with impact angle from 3.2 projectile masses at vertical incidence to 1.9 projectile masses at $30^{\circ}$. The mass of projectile-contaminated pieces exceeds the projectile mass because contaminated pieces are mixtures of the target and projectile.

Figure 5 divides the projectile-contaminated pieces into two groups: those recovered inside the crater and those recovered beyond the rim. The two MFDs evolve complimentarily with impact angle. For example, $>90 \%$ of the recovered projectile-contaminated pieces remained inside the crater at vertical incidence, and a few large pieces dominate the MFD. Outside the crater, in contrast, the MFD for vertical impacts is flat. At $60^{\circ}$ nearly equal masses of projectilecontaminated pieces were recovered inside the crater and beyond the rim. The MFD of pieces inside the crater at $60^{\circ}$ is somewhat shallower than the MFD of projectile-contaminated pieces from the same location in the $90^{\circ}$ experiment. Conversely, the mass-frequency distribution of 
181 pieces beyond the crater rim is steeper at $60^{\circ}$ than at $90^{\circ}$. As impact angle decreases to $45^{\circ}$ and $18230^{\circ}$, the majority of projectile-contaminated pieces are retained beyond the rim (e.g., $\sim 70$ wt. $\%$ 183 beyond the rim at $30^{\circ}$ ). The MFD of projectile-contaminated pieces inside the crater at these 184 angles is relatively flat, but the distribution of pieces beyond the rim steepens with decreasing 185 impact angle. The apparent dearth of projectile-contaminated pieces retained inside the crater at $18645^{\circ}$ relative to the $30^{\circ}$ experiment is compensated by the larger mass of pieces that was 187 recovered beyond the crater rim.

\subsection{Chemical mixing between projectile and target.}

\subsubsection{Aluminum projectiles.}

Spatially distinct metal-rich and silicate-rich regions indicate melt immiscibility (Figure

6). This important observation demonstrates that parts of the aluminum impactor melted during

193 the impact event. Prior work on the $\mathrm{Al}_{2} \mathrm{O}_{3}-\mathrm{SiO}_{2}$ binary system by MacDowell and Beall (1969)

194 revealed a metastable immiscibility gap buried beneath the mullite + liquid and mullite + quartz 195 two phase fields. The gap spans $\sim 10$ to $50 \mathrm{~mol} \% \mathrm{Al}_{2} \mathrm{O}_{3}$, a range of compositions that is entirely 196 plausible based on the microprobe compositions reported in Table 5. Hence, phase equilibria in 197 the $\mathrm{Al}_{2} \mathrm{O}_{3}-\mathrm{SiO}_{2}$ binary system provide a thermodynamic basis for our claim of metal-silicate 198 immiscibility. Metal-silicate immiscibility may be relevant to impacts of metallic projectiles into 199 the Vestan regolith. The extent of immiscibility depends on the enthalpy of mixing of the 200 metallic impactor and silicate regolith. 
excess silicon in the aluminum alloy. The silicate in analyses (2) and (3) of Figure 7B shows depressed $\mathrm{SiO}_{2}$ and elevated $\mathrm{Al}_{2} \mathrm{O}_{3}$ by a few weight percent, along with elevated $\mathrm{CuO}$ in the case of region (3) of Figure 7B. This indicates limited incorporation of the projectile into the silicate melt.

BSE-bright blebs painting the front of the melt zone in Figure 7C are relicts of the projectile. Textures indicate many of these are melted, such as the one near analysis (2) in Figure 7C. Others (e.g., the circled piece) may have been deformed in the solid state. Both melted and unmelted projectile relics were observed in many breccia pieces recovered from this experiment. Microprobe analysis of region (1) in Figure 7C documents completely uncontaminated pumice melt, whereas analysis (2) in Figure 7C again indicates limited incorporation of silicon from the target into regions of melted projectile. Textures in projectile-contaminated pieces produced by the vertical impact of an aluminum projectile into a pumice target indicate that silicate-metal melt immiscibility and shear associated with penetration control projectile-target mixing in the time between the onset of melting and quenching.

\subsubsection{Basalt projectiles.}

Experiments with basalt projectiles reveal dynamic, but highly heterogeneous, projectiletarget mixing during the short time between melting and quenching. Mixing happens between melts of both target and projectile, as well as between melts from the target and solid projectile clasts. For example, the projectile-contaminated pieces in panels (A) and (B) of Figure 8 include basaltic clasts that clearly did not melt. Panel (A) shows several fragmented, relict clasts in the glassy melted portion of projectile-contaminated pieces recovered inside the crater. The clasts preserve relict mineral grains. Microprobe analyses at location (4) in panel 8B, for example, 
227 indicate feldspar. All of the solid basalt clasts seen in the breccias were fractured, presumably

228 during impact. The basaltic clast is rimmed by a thin layer of vesicular melt composed primarily 229 of target pumice (Fig. 8B).Vesicles appear more abundant in the rim than in the rest of the glass, 230 though vesicles are ubiquitous throughout the melt zone. In some places, the rimming melt is 231 slightly enriched in impactor-derived elements (e.g., analysis (3) of panel 8B). Several tens to 232 hundreds of microns away from the encased clast, the melt is essentially pure pumice, with no 233 contamination by the impactor (e.g., analysis (1) and (2) of panel B).

Panels $\mathrm{C}$ and D of Figure 8 emphasize the heterogeneity of mixing. Although mixing among the melted target and projectile is more extensive in this case, as evidenced by variable BSE brightness, even mixed melts remain heterogeneous. Projectile-contaminated melt zones

237 border essentially uncontaminated material (e.g., analyses 8D (1) and 8F (2)). Analyses (1), (3), 238 and (4) in panel 8F indicate that different parts of the glass are associated with elements that 239 derive from the same mineral phase in the projectile. Al and $\mathrm{Na}$, for example, are present in 240 relatively high amounts in analysis (4), but these elements are never paired in high abundance 241 with, for example, Fe or Mg (e.g., analyses 8D (2) and 8F (3)). Consequently, spatial variations

242 in melt composition reflect the mineralogy of the basalt projectile. Mixing and chemical 243 diffusion within the melt cannot homogenize even grain-scale compositional heterogeneity 244 during these experiments

\subsection{Summary of Results.}

Projectile-contaminated pieces consist of a melt-matrix breccia adhered to shockcompressed target pumice. The melt hosts the projectile component, which consists of both melted projectile blebs and relict clasts. Several massive projectile-contaminated pieces 
250 dominate the mass-frequency distributions, but the spatial distribution of these pieces (i.e., inside

251 the crater or beyond the rim) and the shape of the mass-frequency distributions vary with impact

252 angle.

253 The mass of the projectile remaining in or near the crater ranges from $72 \%$ at vertical

254 incidence to $7 \%$ for a $30^{\circ}$ impact. When the probability distribution of impact angles is

255 accounted for, an average of $17 \mathrm{wt} . \%$ of the projectile remains in or near the crater during

256 impacts at 4.5 to $5 \mathrm{~km} \mathrm{~s}^{-1}$. Chemical analyses reveal that mixing between projectile and target is

257 vigorous, but the short timescales preclude homogenization.

258

259

4. Discussion

260

4.1. Uncertainties in absolute retained mass.

Several factors could introduce uncertainties in the calculated projectile retention

262 efficiencies. First, the sample recovery strategy did not recover the very finest projectile-

263 contaminated pieces. Some portion of the projectile is therefore not accounted for in this

264 analysis. However, high-speed cameras viewed the impact experiment from multiple angles and

265 confirmed that no large projectile-contaminated pieces escaped recovery. Uncertainties

266 associated with incomplete sample recovery will therefore be small.

267 Second, contamination by a third compositional endmember could introduce uncertainties

268 into the $X_{\text {Bas }}$ values calculated using the chemical mixing model (section 3.1). However, all of

269 the mixing trends are highly linear, which indicates that this source of error is also quite small.

270 The error bars shown in Figure 3 and listed in Table 4 represent this source of uncertainty.

Third, in some experiments, projectile-contaminated pieces from only one location-

272 either inside the crater or beyond the rim-were analyzed with ICP-AES due to the small mass 
273 of projectile-contaminated pieces recovered from the other location. Projectile-contaminated

274 pieces from the other location were analyzed solely in thin section. This necessary approach

275 introduces uncertainties into the projectile retention efficiencies calculated from these

276 experiments. Nevertheless, these uncertainties are quite small. In all cases, the mass of pieces

277 analyzed solely in thin section was small compared to the mass of projectile-contaminated pieces

278 analyzed by ICP-AES. Furthermore, microprobe analyses revealed that the potential projectile

279 contribution from these pieces is negligible (<1 wt. \%) for experiments at $45^{\circ}$ and $90^{\circ}$. Applying

280 the two-component mixing model (section 3.1) to microprobe data from these experiments

281 (Figure 9, Table 6) yielded statistically insignificant results in both cases $\left(P>0.05 ; r^{2}<0.10\right)$.

282 For the $30^{\circ}$ experiment, the two-component mixing model indicates that $X_{\text {Bas }}$ in the thin section

283 is comparable to the bulk $X_{B a s}$ calculated from ICP-AES data. However, the mass of projectile-

284 contaminated pieces from the $30^{\circ}$ experiment that were analyzed solely in this section is so small

285 that these pieces contribute at most $2 \%$ of a projectile mass. These uncertainties are comparable

286 to the two-sigma uncertainties in the two-component mixing model. Therefore, these

287 uncertainties do not affect conclusions drawn from these data.

288

289

\subsection{Spatial variation and impact angle effects.}

290 As impact becomes increasingly oblique, projectile-contaminated pieces disperse

291 downrange beyond the crater rim. Yet, projectile-contaminated pieces recovered within the crater

292 for $30^{\circ}$ impacts contain the highest abundance of relict projectile clasts (compare Figures 8A and

$2938 \mathrm{~B}$ with $8 \mathrm{C}-8 \mathrm{~F})$. Prior experiments indicate that large fractions of the projectile survive at lower

294 impact angles but disperse at high speeds downrange (Schultz and Gault, 1990). Survival was

295 attributed to the lower peak pressures in the projectile while retaining the initial impactor 
momentum. The spatial distribution of fragments hitting witness plates downrange indicates that they originated from the top and sides of the projectiles (Schultz and Sugita, 1997). Other portions (near contact and inside), however, undergo intense heating and comminution. The experiments here indicate that the lower peak pressures also ensure survival of intact projectile clasts inside the crater, with the clasts encased in target-derived melt. These fragments, however, likely come from those portions uprange from the contact zone where peak pressures would have been reduced, similar to the distribution of damage produced in spheres by oblique impacts (Schultz and Crawford, 2011; Stickle and Schultz, 2015). While we basalt clasts were always fractured, unmelted aluminum pieces were unfractured. At lower impact speeds and reduced shock pressures, unfractured basalt clasts would likely also be found.

\subsection{Implications for Vestan dark material.}

Substantial projectile retention happens for impacts at typical main belt encounter speeds (e.g., $4.5-5 \mathrm{~km} \mathrm{~s}^{-1}$ ) (O’Brien and Sykes, 2011). This result is independent of results from numerical impact models (e.g., Svetsov, 2011; Turrini et al., 2014; Turrini and Svetsov, 2014). Extensive projectile delivery and retention do not require low-probability very low-speed impacts (e.g., $<2 \mathrm{~km} \mathrm{~s}^{-1}$ ), as previously suggested by Reddy et al. (2012). Hence, the delivery of material from both stony and metallic impactors to Vesta should be ubiquitous, not an exceptional event. Nevertheless, lower impact speeds would enhance projectile retention. Carbonaceous chondrite-like impactors are likely less competent and more porous than the basalt projectiles used here. Nevertheless, the retention of carbonaceous chondrite impactors is likely similar to what is observed for basalt projectiles. Projectiles of both types will fail in a brittle manner. Fragment sizes in both cases will be influenced by the critical flaw size of the 
material. Future studies will assess how projectile porosity affects the projectile retention efficiencies measured in hypervelocity lab experiments.

While experiments capture some of the detailed physical and chemical processes that current numerical impact simulations cannot, experiments are by necessity small in scale. The strategy is to isolate a process and then use scaling relationships, comparisons with numerical models, and the planetary cratering record to extrapolate the results of laboratory impact experiments to planetary scales. Although current impact models do not accurately represent key factors in the projectile delivery process, comparing large-scale numerical impact simulations with small-scale experiments helps constrain how results from experiments scale. Turrini and Svetsov (2014) provide the numerical impact simulations that most closely match the impact speeds used in these experiments. The experiments reported in the study described in this paper reveal that $14 \pm 4 \%$ of the mass of a basalt projectile is retained in or near the crater for a $45^{\circ}$ impact at $\sim 4.5 \mathrm{~km} \mathrm{~s}^{-1}$. Linearly interpolating data from model runs by Turrini and Svetsov (2014) that included friction indicates a projectile retention efficiency of $17 \mathrm{wt} . \%$ for water ice projectiles impacting at $\sim 5 \mathrm{~km} \mathrm{~s}^{-1}$ at an angle of $45^{\circ}$. Note, however, that numerical impact models with ice projectiles, such as those used by Turrini and Svetsov (2014) consistently underestimate projectile retention for silicate projectiles, like those used in experiments (Bruck Syal and Schultz, 2014). Experiments assess projectile retention in and within a few crater radii of the impact crater while numerical models assess projectile retention with respect to the escape velocity of the target object: the two techniques assess projectile retention over local and global scales, respectively. Given that difference, it makes sense that experimental studies would predict lower projectile retention efficiencies than numerical models. The local projectile 
341 retention, however, is relevant to the distribution of the projectile component in and near the 342 crater.

While these experiments reveal that impactor delivery to Vesta is viable, this study does 344 not directly link dark material to impact-delivered carbonaceous chondrites. Other mechanisms 345 could create dark material deposits on Vesta. For example, terrestrial impact melt breccias 346 formed in porous loess deposits are dark without being heavily contaminated by the projectile 347 (Schultz et al., 2006; Schultz and Mustard, 2004; Schultz and Lianza, 1992). However, reduced 348 impact melt production on Vesta compared to Earth (due to the lower impact speeds) lessens the 349 likelihood that impact melts are the cause of dark material on Vesta. Other explanations for dark 350 material on Vesta, including grain size variations, the presence of opaque mineral phases or metal, shock processing, or basaltic lava flows have been thoroughly assessed by other studies

352 (McCord et al., 2012; Reddy et al., 2012; de Sanctis et al., 2012; Palomba et al., 2014; Jaumann 353 et al., 2014). Small dark deposits without associated hydration signatures could be related to 354 these other darkening processes (e.g., Palomba et al., 2014). Nevertheless, the correlation 355 between albedo and hydration, the detection of serpentine in dark material (Nathues et al., 2014), 356 and the presence of carbonaceous chondrite clasts in howardites (Zolensky et al., 1996; Herrin et 357 al., 2011) provides strong evidence that carbonaceous chondrite-like material is the agent 358 responsible for dark material on Vesta. Furthermore, the concentration of $\mathrm{H}$ measured in the 359 Vestan regolith by Prettyman et al. (2012) matches the abundance of $\mathrm{H}$ in carbonaceous 360 chondrites found in howardites. The $\mathrm{H}$ is present as $\mathrm{OH}$ in the Vestan regolith, just as it is in 361 carbonaceous chondrites (De Sanctis et al., 2012). Finally, the spectra of dark regions match the 362 spectra of mixtures of carbonaceous chondrites and eucrites (Reddy et al., 2012). This study reveals that the mechanism proposed by Prettyman et al. (2012), McCord et al. (2012), Reddy et 
al. (2012), and de Sanctis et al. (2012) for getting carbonaceous chondrite-like on the surface of

Vesta (namely impactor delivery) is viable. Hence, the results of this study and earlier work strongly support an impact origin for dark material on Vesta.

Experiments predict that other impactors (e.g., metallic and ordinary chondrite) also should contaminate the Vestan regolith. The presence of multiple types of carbonaceous chondrites (Zolensky et al., 1996) and iron meteorite (Hewins, 1979) in howardites, as well as other rare, but diverse, exogenous inclusions observed in howardites by Lorenz et al. (2001) provide direct evidence for a wide array of meteoritic material in the Vestan and/or Vestoid regolith. A key question is whether the high abundance of carbonaceous chondrite clasts in howardites relative to other impactors reflects differing fluxes, different projectile retention efficiencies, or both. The textural differences between projectile-contaminated pieces recovered from experiments with basalt projectiles and aluminum projectiles indicate that differences in either projectile retention efficiency or the breakdown of the delivered projectile component through time may depend on impactor material properties.

To date, spectral data from the Dawn mission to Vesta have not convincingly revealed evidence for impactor contamination, other than carbonaceous chondrites, perhaps because carbonaceous chondrites leave dark, hydrous traces that are relatively easy to identify with VNIR data. Le Corre et al. (2015) may have recently detected exogenic olivine around Arruntia and Bellicia craters. However, searching for a specific concordance between the distributions of exogenous clasts in the howardites, Dawn data, and predictions based on the impact flux and projectile retention efficiencies may not be entirely relevant. The howardites most recently derived from the Vestoids and may have had a different contamination history after being excavated from Vesta. 


\section{Conclusions}

Based on our experiments, parts of many of the projectiles that impact Vesta are delivered to and retained in the regolith. Impact delivery is therefore a viable origin for Vestan dark material. The results of this study indicate that the "continuous flux" scenario proposed by McCord et al. (2012) and Turrini et al. (2014) best describes the accumulation of dark material on Vesta through time. The continual flux of impactors of all types - not merely dark impactors - will produce projectile relics, projectile-contaminated glasses, and projectile-bearing breccias. These projectile-contaminated materials should accumulate in the regolith through time. Although much of the projectile component would likely initially be hosted in breccias, continuing bombardment would comminute the impact products, slowly mixing the projectile component into the bulk Vestan regolith. Nevertheless, the continuous flux hypothesis is consistent with localized deposits of projectile-rich material (e.g., dark, hydrous material for carbonaceous chondrite-like impactors) around and within craters. These localized deposits represent a projectile component that has not yet been reworked and gardened sufficiently to become spatially diffuse. Craters at or above the equilibrium crater diameter can preserve localized deposits of impactor material.

Previous authors have suggested that stochastic events are needed to explain the dark material on Vesta (e.g., Reddy et al., 2012). The results of experiments cannot rule out a stochastic event, such as the large, very low velocity collision that Reddy et al. (2012) invoke for Veneneia, delivered significant quantities of dark projectile material. Nevertheless, experiments reveal that most of the impacts that have occurred on Vesta should have delivered a projectile component. A large event involving a dark impactor might possibly help explain the hemispherical albedo dichotomy on Vesta (Reddy et al., 2012), but based on our experiments the 
410 impact conditions needed for this hypothesis to work are likely not as restrictive as Reddy et al.

411 (2012) suggest. Large impact events are a natural part of the continuous flux scenario (Turrini et

412 al., 2014). These experiments indicate that the perpetual bombardment by objects ranging from

413 massive to miniscule gives rise to a projectile component on Vesta. Rare events are not a

414 necessary condition for impactor delivery. Not every impact will contribute a projectile

415 component to the surface, but the projectile component imparted by past impacts likely litters the 416 Vestan regolith.

417 Impactor delivery and retention is not unique to Vesta. Environments with relatively low

418 impact speeds (a few $\mathrm{km} \mathrm{s}^{-1}$ ) and porous targets favor capturing projectiles within the target.

419 Diverse exogenous clasts in the Almahata Sitta meteorite (Goodrich et al., 2015) speak to the 420 importance of projectile survival on multiple meteorite parent bodies, not solely Vesta. The 421 regoliths of other large bodies in the main belt and in other environments with impact speeds of a 422 few $\mathrm{km} \mathrm{s}^{-1}$ are likely littered with projectile relicts, projectile-bearing melt matrix breccias, and 423 projectile-rich impact melts.

\section{Acknowledgements}

We thank Joseph Boesenberg, David Murray, Joe Orchardo, Anthony McCormick,

427 Rebecca Greenberger, and Bill Collins for help with sample preparation and analysis.

428 Stimulating conversations with Jerry Delany and Axel Whittmann fostered a deeper

429 understanding of the howardites. We especially thank the technical crew of the NASA Ames

430 Vertical Gun Range, whose efforts enabled the experiments that underpin this work. This work is

431 supported by NASA grant NNX13AB75G and a National Science Foundation Graduate

432 Research Fellowship under NSF grant DGE-1058262. 


\section{References}

Allegre C. J., Schiano, P., Lewin, E., 1995, Differences between oceanic basalts by multitrace element ratio topology, Earth and Planetary Science Letters, 129, 1 - 12.

Artemieva, N. and Pierazzo, E., 2011, The Canyon Diablo impact event: 2. Projectile fate and target melting upon impact, Meteoritics \& Planetary Science, 46, 805 - 829.

Bland, P. A., Artemieva, N. A., Collins, G. S., Bottke, W. F., Bussey, B. J., Joy, K. H., 2008, Asteroids on the Moon: Projectile survival during low velocity impact, Lunar and Planetary Science Conference XXXIX, abstract no. 2045.

Bruck Syal, M. and Schultz, P. H., 2015, Impact delivery of water at the Moon and Mercury, 2015, Lunar and Planetary Science Conference XXXXVI, abstract no. 1680.

Bruck Syal, M., Schultz, P. H., and Riner, M., 2015 Painting Mercury Black with Cometary Carbon, Nature Geoscience, 8, 352 - 356.

Cantagrel, J.-M., Didier, J., Gourgaud, A., 1984, Magma mixing: Origin of intermediate rocks and "enclaves" from volcanism to plutonism, Physics of the Earth and Planetary Interiors, 35, $63-76$.

Daly, R. T. and Schultz, P. H., 2013, Experimental studies into the survival and state of the projectile, Lunar and Planetary Science Conference XXXXIV, abstract no. 2240.

de Sanctis, M. C., Combe, J. P., Ammannito, E., Palomba, E., Longobardo, A., McCord, T. B., Marchi, S., Capaccioni, F., Capria, M. T., Mittlefehldt, D. W., Pieters, C. M., Sunshine, J., Tosi, F., Zambon, F., Carraro, F., Fonte, S., Frigeri, A., Magni, G., Raymond, C. A., Russell, C. T., and Turrini, D., 2012, Detection of widespread hydrated materials on Vesta by the VIR imaging spectrometer on board the Dawn mission, Astrophysical Journal Letters, 758, L36. 
Devine, J. D., Gardner, J. E., Brack, H. P., Layne, G. D., and Rutherford, M. J., 1995, Comparison of microanalytical methods for estimating $\mathrm{H}_{2} \mathrm{O}$ contents of silicic volcanic glasses, American Mineralogist, 80, 319 - 328.

Gault, D. E. and Wedekind, J. A., 1978, Experimental studies of oblique impact, Proceedings of the $9^{\text {th }}$ Lunar and Planetary Science Conference, p. $3843-3875$.

Gilbert, G. K., 1893, The Moon's face: A study of the origin of its features, Bulletin of the Philosophical Society of Washington, D. C., 12, 241 - 292.

Goodrich, C. A., Hartmann, W. K., O’Brien, D. P., Weidenschilling, S. J., Wilson, L., Michel, P., and Jutzi, M., 2014, Origin and history of urelitic material in the solar system: the view from asteroid $2008 \mathrm{TC}_{3}$ and the Almahata Sitta meteorite, Meteoritics and Planetary Science, DOI: 10.1111/maps.12401 (currently in Early View).

Herrin, J. S., Zolensky, M. E., Cartwright, J. A., Mittlefehldt, D. W., Ross, D. K., 2011, Carbonaceous chondrite-rich howardites: The potential for hydrous lithologies on the HED parent, Lunar and Planetary Science Conference XXXXII, abstract no. 2806.

Hewins, R. H., 1979, Composition and origin of metal in howardites, Geochimica Et Cosmochimica Acta, 43, 1663 - 1673.

Jaumann, R., Nass, A., Otto, K., Krohn, K., Stephan, K., McCord, T. B., Williams, D. A., Raymond C. A., Blewett, D. T., Hiesinger H., Yingst, R. A., De Sanctis, M. C., Palomba, E., Roatsch, T., Matz K.-D., Preusker, F., Scholten, F., Russell, C. T., 2014, The geological nature of dark material on Vesta and implications for the subsurface structure, Icarus, $240,3-19$. 
477 Le Corre, L., Reddy, V., Sanchez, J., Dunn, T., Cloutis, E. A., Izawa, M. R., Mann, P., Nathues,

478

479

480

481

482

483

484

485

486

487

488

489

490

491

492

493

494

495

496

497

498

499

A., 2015, Exploring exogenic sources for the olivine on asteroid Vesta, Lunar and Planetary Science Conference XXXXVI, abstract no. 1107.

Lorenz, C., Nazarov, M., Kurat, G., Brandstaetter, F., Ntaflos, T., 2001, Clast population and chemical bulk composition of the Dhofar 018 howardite, Lunar and Planetary Science Conference XXXII, abstract no. 1778.

Macdowell, J. F. and Beall, G. H., 1969, Immiscibility and crystallization in $\mathrm{Al}_{2} \mathrm{O}_{3}-\mathrm{SiO}_{2}$ glasses, Journal of the American Ceramic Society, 52, 1, 17-25.

McCord, T. B., Li, J. Y., Combe, J. P., McSween, H. Y., Jaumann, R., Reddy, V., Tosi, F., Williams, D. A., Blewett, D. T., Turrini, D., Palomba, E., Pieters, C. M., de Sanctis, M. C., Ammannito, E., Capria, M. T., Le Corre, L., Longobardo, A., Nathues, A., Mittlefehldt, D. W., Schroeder, S. E., Hiesinger, H., Beck, A. W., Capaccioni, F., Carsenty, U., Keller, H. U., Denevi, B. W., Sunshine, J. M., Raymond, C. A., and Russell, C. T., 2012, Dark material on Vesta from the infall of carbonaceous volatile-rich material, Nature, 491, 83 - U93.

Murray, R. W., Miller, D. J., and Kryc, K. A., 2000. Analysis of major and trace elements in rocks, sediments, and interstitial waters by inductively coupled plasma-atomic emission spectrometry (ICP-AES). ODP Tech. Note, 29. doi:10.2973/odp.tn.29.2000

Nathues, A., Hoffmann, M., Cloutis, E. A., Schäfer, M., Reddy, V., Christensen, U., Sierks, H., Thangjam, G. H., Le Corre, L., Mengel, K., Vincent, J.-B., Russell, C.T., Prettyman, T., Schmedemann, N., Kneissl, T., Raymond, C., Gutierrez-Marques, P., Hall, I., Büttner, I., 2014, Detection of serpentine in exogenic carbonaceous chondrite material on Vesta from Dawn FC data, Icarus, 239, 222 - 237. 
O’Brien, D. P. and Sykes, M. V., 2011, The origin and evolution of the asteroid beltimplications for Vesta and Ceres, Space Science Reviews, 163, 41 - 61.

Ong, L., Asphaug, E. I., Korycansky, D., and Coker, R. F., 2010, Volatile retention from cometary impacts on the Moon, Icarus, 207, 578 - 589.

Palomba, E., Longobardo, A., De Sanctis, M. C., Zambon, F., Tosi, F., Ammannito, E., Capaccioni, F., Frigeri, A., Capria, M. T., Cloutis, E. A., Jaumann, R., Combe, J.-P., Raymond, C. A., Russell, C. T., 2014, Composition and mineralogy of dark material units on Vesta, Icarus, 240, $58-72$.

Pierazzo, E. and Chyba, C. F., 2002, Cometary delivery of biogenic elements to Europa, Icarus, $157,120-127$.

Prettyman, T. H., Mittlefehldt, D. W., Yamashita, N., Lawrence, D. J., Beck, A. W., Feldman, W. C., McCoy, T. J., McSween, H. Y., Toplis, M. J., Titus, T. N., Tricarico, P., Reedy, R. C., Hendricks, J. S., Forni, O., Le Corre, L., Li, J.-Y., Mizzon, H., Reddy, V., Raymond, C. A., and Russell, C. T., 2012, Elemental Mapping by Dawn Reveals Exogenic H in Vesta's Regolith, Science, 338, 242 - 246.

Reddy, V., Le Corre, L., O'Brien, D. P., Nathues, A., Cloutis, E. A., Durda, D. D., Bottke, W. F., Bhatt, M. U., Nesvorny, D., Buczkowski, D., Scully, J. E. C., Palmer, E. M., Sierks, H., Mann, P. J., Becker, K. J., Beck, A. W., Mittlefehldt, D., Li, J.-Y., Gaskell, R., Russell, C. T., Gaffey, M. J., McSween, H. Y., McCord, T. B., Combe, J.-P., and Blewett, D., 2012, Delivery of dark material to Vesta via carbonaceous chondritic impacts, Icarus, $221,544-559$.

Russell, C. T., Raymond, C. A., Coradini, A., McSween, H. Y., Zuber, M. T., Nathues, A., de Sanctis, M. C., Jaumann, R., Konopliv, A. S., Preusker, F., Asmar, S. W., Park, R. S., 
Gaskell, R., Keller, H. U., Mottola, S., Roatsch, T., Scully, J. E. C., Smith, D. E., Tricarico, P., Toplis, M. J., Christensen, U. R., Feldman, W. C., Lawrence, D. J., McCoy, T. J., Prettyman, T. H., Reedy, R. C., Sykes, M. E., and Titus, T. N., 2012, Dawn at Vesta: Testing the Protoplanetary Paradigm, Science, 336, 684 - 686.

Schultz, P. H. and Crawford, D. A., 2011, Origin of nearside structural and geochemical anomalies on the Moon, Geological Society of America Special Paper 477, 141 - 159.

Schultz, P. H. and Stickle, A. M., 2011, Arrowhead craters and tomahawk basins: Signatures of oblique impacts at large scales, Lunar and Planetary Science Conference XXXXII, abstract no. 1608 .

Schultz, P. H. and Sugita, S., 1997, Fate of the Chicxulub impactor, Lunar and Planetary Science Conference XXVIII, abstract no. 1792.

Stickle, A. M. and Schultz, P. H., 2015, Subsurface failure in spherical bodies: A formation scenario for linear troughs on Vesta's surface, Icarus, 247, 18-34.

Svetsov, V., 2011, Cratering erosion of planetary embryos, Icarus, 214, 316 - 326.

Turrini, D. and Svetsov, V., 2014, The formation of Jupiter, the Jovian Early Bombardment, and the delivery of water to the asteroid belt: The case of (4) Vesta, Life, 4, 4-34.

Turrini, D., Combe, J. P., McCord, T. B., Oklay, N., Vincent, J.-B., Prettyman, T. J., McSween, H. Y., Consolmagno, G. J., De Sanctis, M. C., Le Corre, L., Longobardo, A., Palomba, E., Russell, C. T., 2014, The contamination of the surface of Vesta by impacts and the delivery of the dark material, Icarus, 240, 86 - 102.

Zolensky, M. E., Weisberg, M. K., Buchanan, P. C., and Mittlefehldt, D. W., 1996, Mineralogy of carbonaceous chondrite clasts in HED achondrites and the Moon, Meteoritics \& Planetary Science, 31, 518 - 537. 

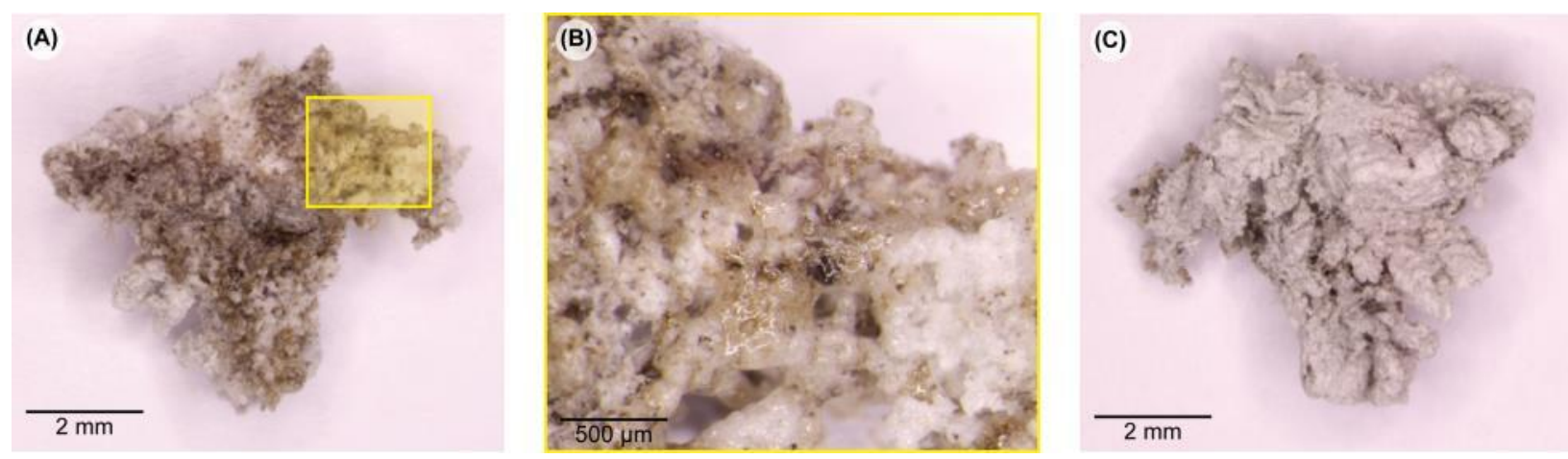

Figure 1. A typical projectile-contaminated piece. (A) The glassy side consists of dark

blebs of melted projectile, along with occasional solid projectile relics, encased in melted target

pumice. This is a region of intense physical and chemical mixing during crater excavation. (B) A

551 zoomed in view of the region inside the yellow box in (A). The glassy sheen indicates melted

552 material. The greenish glass is contaminated by the projectile; the white glass is almost pure

553 pumice melt derived from the target. The dark, oval-shaped inclusion near the center of the

554 micrograph is a solid fragment of the basalt projectile. (C) The other side of the piece consists of

555 shock-compressed target pumice welded to the glassy melt. This piece was recovered near the

556 rim of a crater formed by a $1 / 4$ " basalt sphere impacting a pumice target at $4.93 \mathrm{~km} \mathrm{~s}^{-1}, 60^{\circ}$ from

557 horizontal. 
558

559

560

561 the line gives $X_{\text {Bas }}$, the mass fraction of the projectile in the projectile-contaminated pieces. (A)

562 The data for this representative sample are highly linear $\left(r^{2}=0.9963\right)$, consistent with a two-

563 component mixture of the projectile and target. The regression slope $\left(X_{\text {Bas }}=0.19\right)$ indicates that

564 the projectile-contaminated pieces from this experiment are $19 \%$ wt. \% projectile. (B) The data

565 remain highly linear $\left(r^{2}=0.9896\right)$ even when influential $\mathrm{MnO}, \mathrm{TiO}_{2}$, and $\mathrm{P}_{2} \mathrm{O}_{5}$ are removed. The

566 slope of the regression line is unaffected by removing these data.

Figure 2. If projectile-contaminated pieces are mixtures of the basalt projectile and pumice target, then the oxides from each sample will form a line through the origin. The slope of
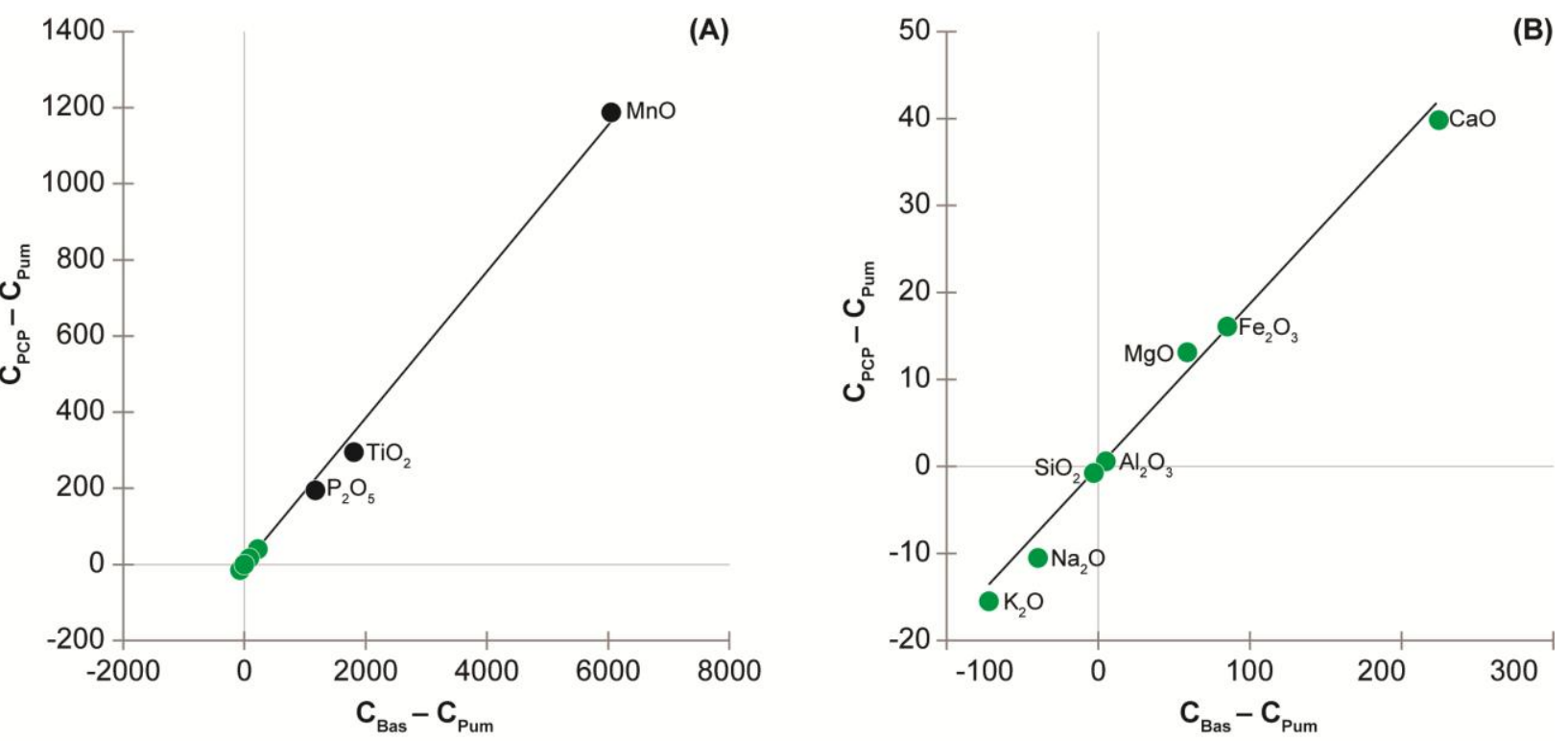

(B) 


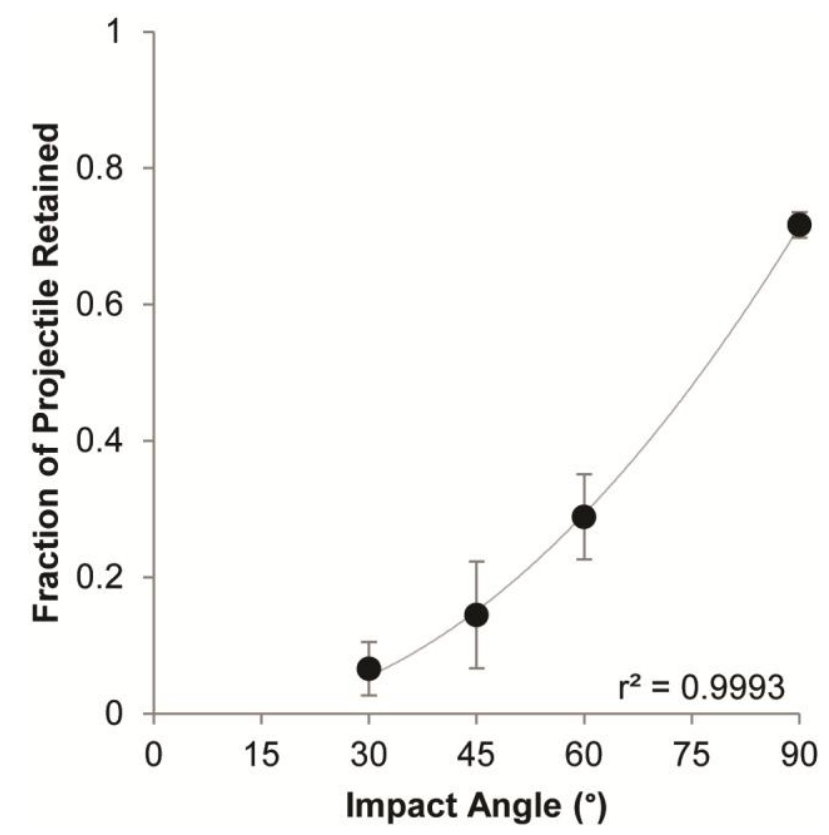

Figure 3. Projectile retention efficiency for basalt projectiles impacting asteroid regolith 569 analogs at $4.5-5 \mathrm{~km} \mathrm{~s}^{-1}$. The gray line is an empirical $2^{\text {nd }}$ order polynomial regression.

570 Integrated over the probability distribution of impact angles, an average of $17 \%$ of the mass of

571 basalt projectiles remains inside or near the crater at these speeds. Error bars reflect two sigma 572 uncertainties. 


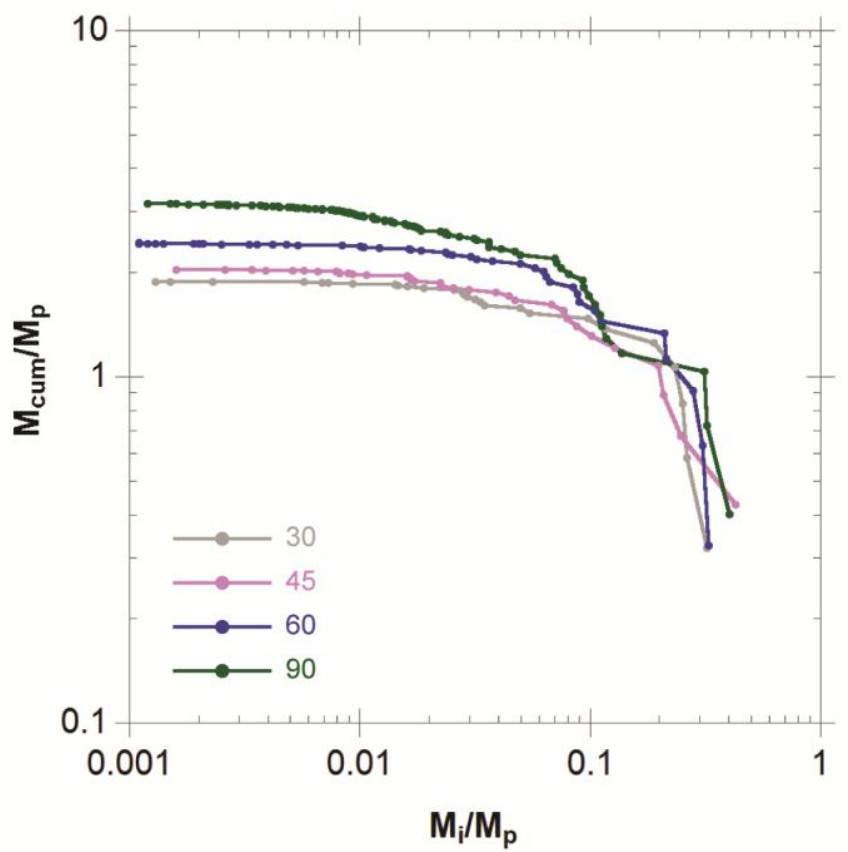

Figure 4. Cumulative mass frequency distributions of projectile-contaminated pieces

575 from impact experiments conducted at a variety of impact angles using basalt projectiles and

576 pumice targets. The $x$-axis is the mass of an individual breccia piece, $M_{i}$, normalized to the

577 original mass of the projectile, $M_{p}$. The $y$-axis, $M_{c u m}$, is the cumulative mass of all pieces with 578 mass $\geq M_{i}$, again normalized to the original projectile mass, $M_{p}$. 


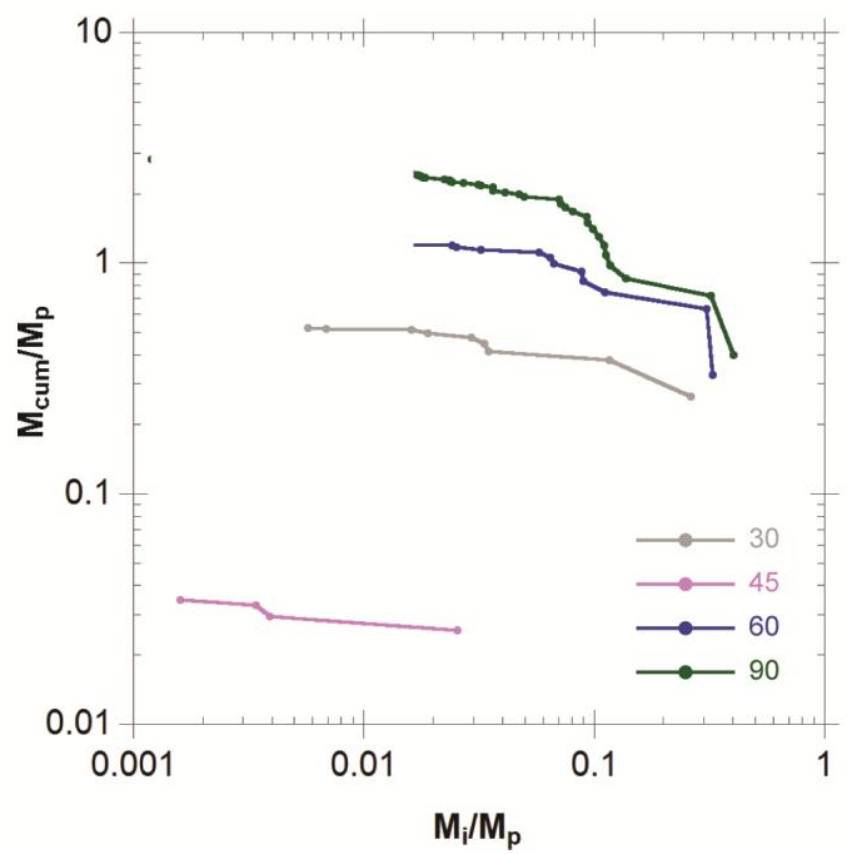

(A) Retained Inside the Crater

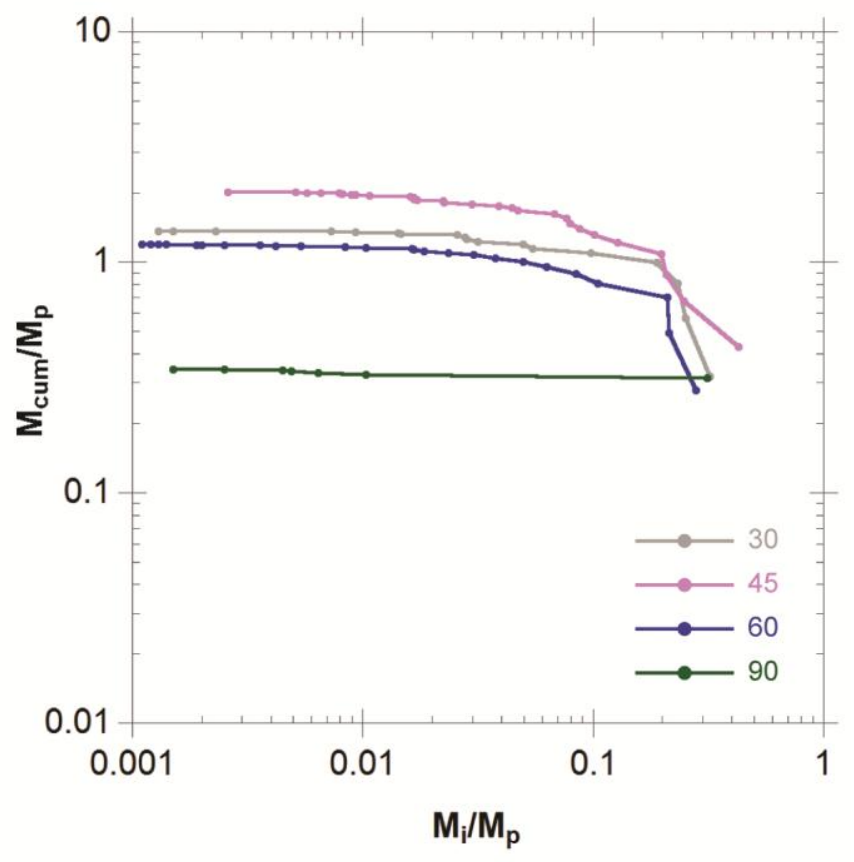

(B) Ejected Beyond the Rim

Figure 5. The cumulative mass-frequency distributions of projectile-contaminated pieces

581 recovered from (A) inside the crater and (B) beyond the rim evolve with impact angle. The mass

582 of pieces retained inside the crater decreases with impact angle; the mass of pieces ejected 
583 beyond the rim increases. In addition, the mass-frequency distribution of projectile-contaminated 584 pieces inside the crater shallows while the MFD of projectile-contaminated pieces beyond the 585 rim steepens as impact become more oblique. See text for a discussion of the $45^{\circ}$ data.

586 


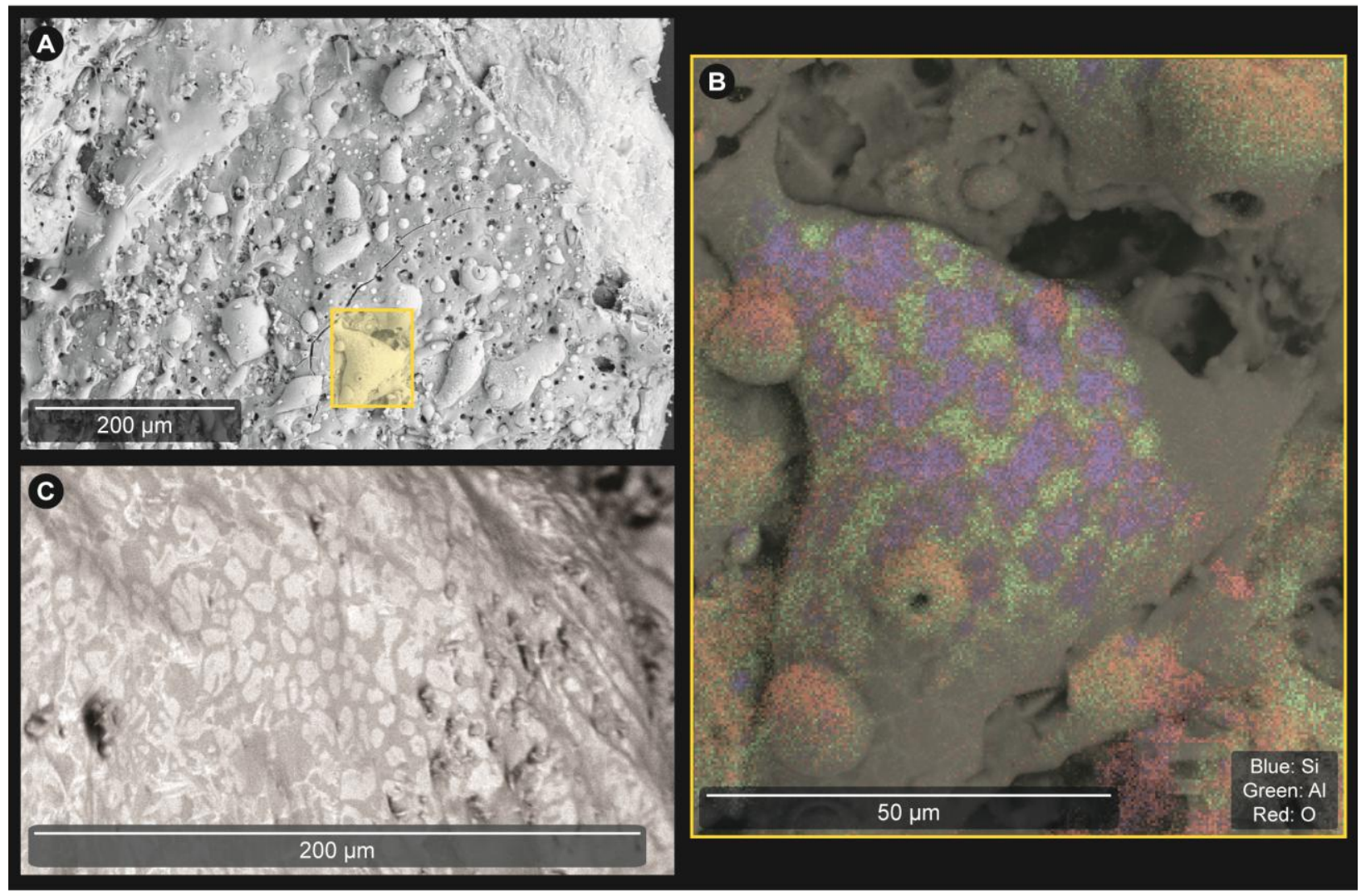

587

Figure 6. SEM/EDS images from projectile-contaminated pieces generated by the vertical impact of an aluminum projectile into a pumice target. (A) Backscatter electron micrograph providing context for the element map in (B). The bead-like features are silicate-rich melt atop a metal substrate. (B) Element map of the area inside the yellow box in (A). Blue is silicon, green is aluminum, and red is oxygen. Silicon and aluminum separate into discrete regions, indicating silicate-aluminum melt immiscibility in the time between melting and quenching. (C) shows another backscatter electron micrograph from a different area of the sample. The texture here also indicates immiscibility. The brighter regions are aluminum (projectile) rich while the darker regions are silicate (target) rich. 

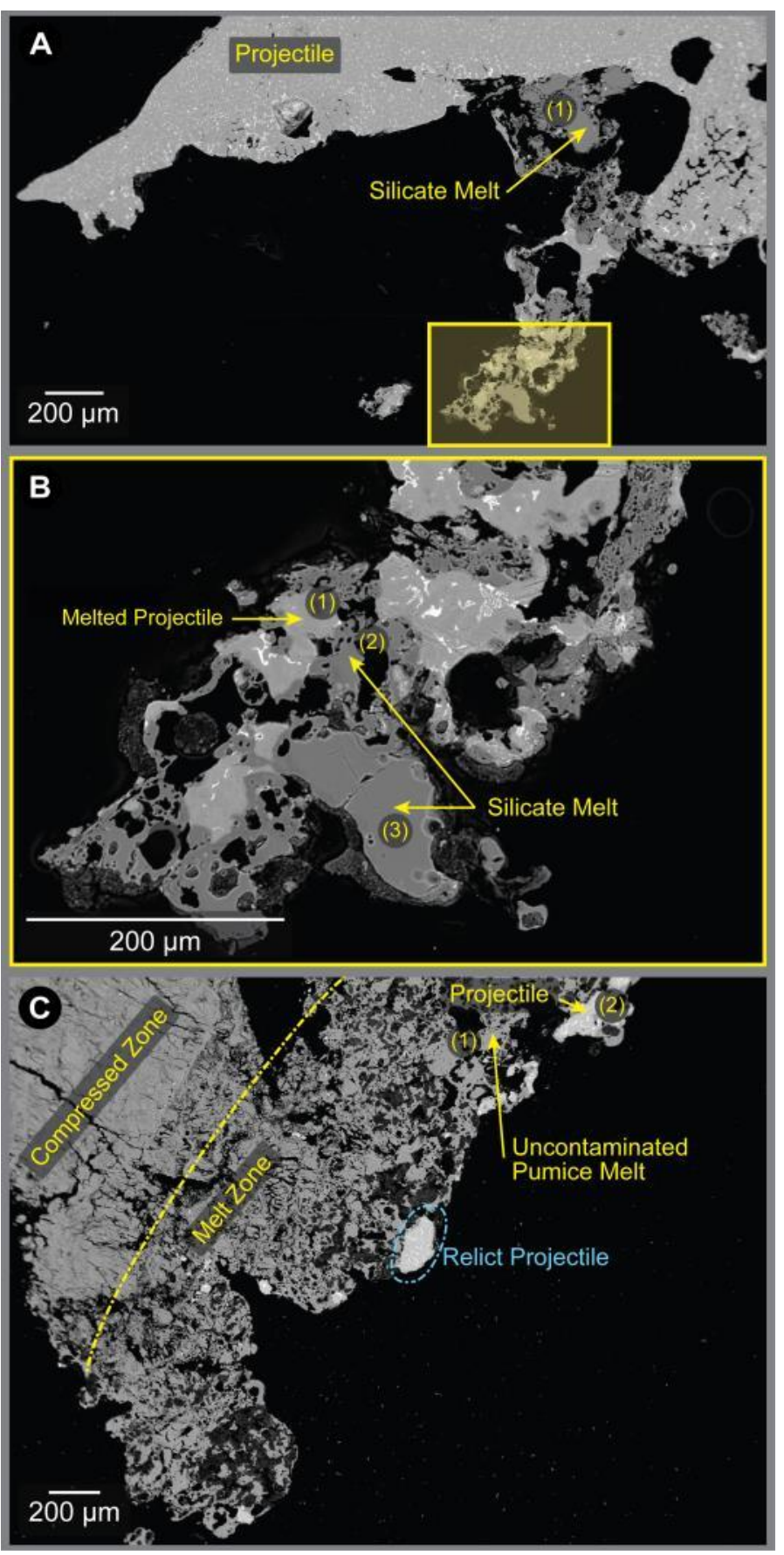

Figure 7. Backscatter electron micrographs of cross sections of two different projectile-

599 contaminated pieces from the same experiment shown in Figure 6. The numbered arrows point to 600 areas of electron microprobe analyses described in the text and listed in Table 5. (A) Silicate and 601 aluminum melts stay in separate areas with sharp boundaries, a texture that indicates very limited 602 mixing. The bright regions in the metal are $\mathrm{Cu}$-rich. (B) Higher magnification view of the area 
603 boxed in yellow in (A). (C) Another projectile-contaminated piece from the same experiment.

604 The melt-zone/compressed zone structure is typical of most projectile-contaminated pieces. The

605 isolated blebs of aluminum projectile lining the front of the melt zone indicate shear was

606 important during formation of projectile-contaminated pieces - even though the impact was

607 vertical. 

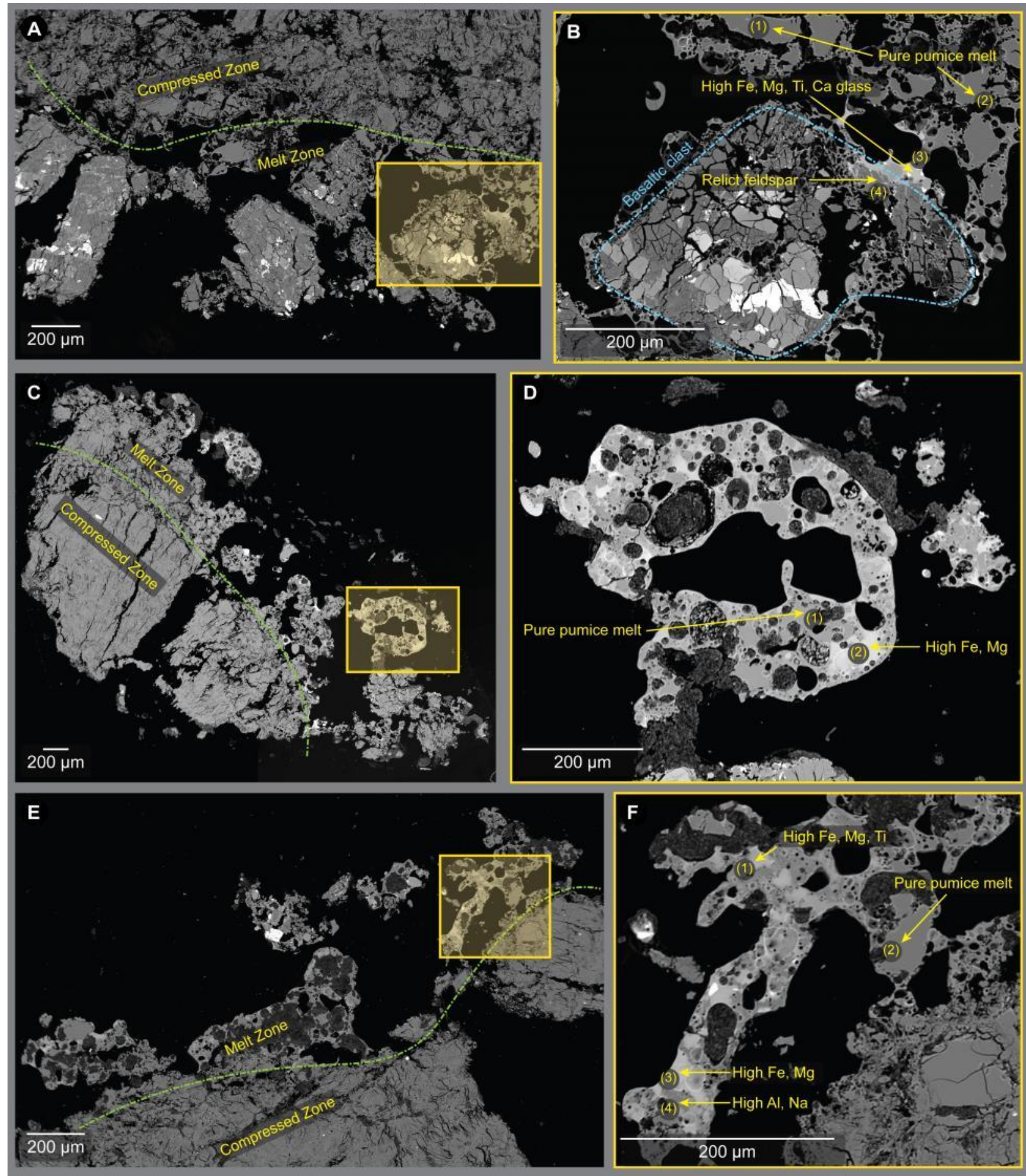

Figure 8. Backscattered electron micrographs of thin sections of projectile-contaminated

610 pieces created by the impact of basalt projectiles into pumice target. (A) and (B) are from a $30^{\circ}$

611 impact; (C) and (D) from a $90^{\circ}$ impact; and (E) and (F) from a $45^{\circ}$ impact. All these projectile-

612 contaminated pieces were recovered inside the crater. Numbered arrows correspond to 
613 microprobe analyses in Table 5. Panels at right are higher magnification views of the regions 614 highlighted with yellow boxes at left. (A) \& (B) show a relict basaltic clast encapsulated by 615 vesicular target melt. In small areas (e.g., B(3)), the projectile has lightly contaminated the 616 rimming melt. $(\mathrm{C})-(\mathrm{F})$ reveal extreme heterogeneity in the melt zone, with uncontaminated 617 target melt (e.g., D(1), F(2)) juxtaposed against melt clearly contaminated by the projectile (e.g., $618 \mathrm{D}(2), \mathrm{F}(1)-\mathrm{F}(4))$. Elements that partition into the same phases are found together (e.g., Al, $\mathrm{Na}$ 619 in $\mathrm{F}(4)$ and $\mathrm{Fe}, \mathrm{Mg}$ in $\mathrm{D}(2)-\mathrm{D}(4)$ ), which indicate that even grain-scale chemical variations 620 cannot be homogenized during the time between melting and quenching in these experiments. 

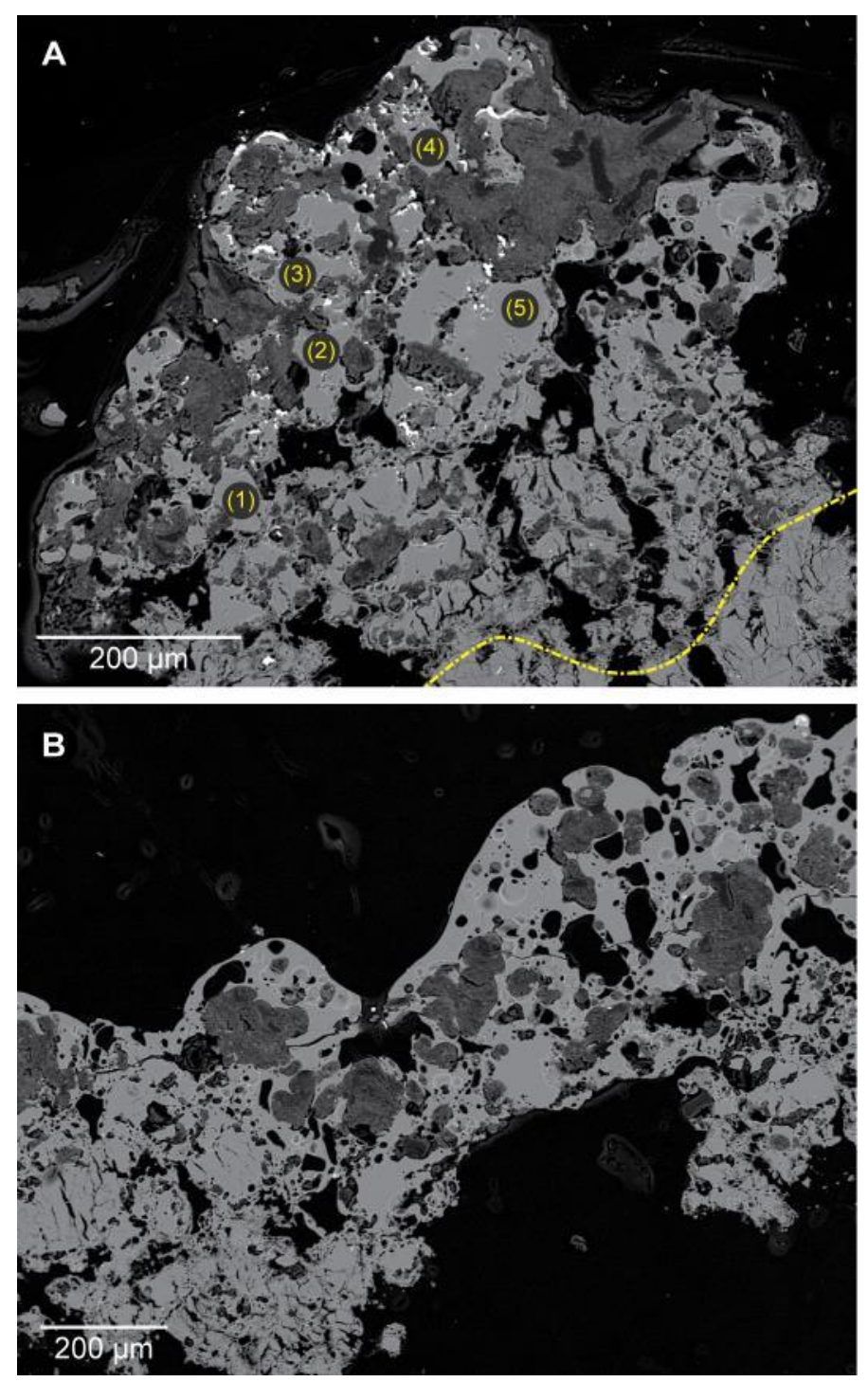

622 Figure 9. Microprobe data reveal that projectile-contaminated pieces set aside for thin sectioning

623 from experiments at $45^{\circ}$ and $90^{\circ}$ contain no chemical traces of the projectile. Hence, unanalyzed

624 fractions of projectile-contaminated pieces do not introduce meaningful uncertainties into the

625 calculated projectile retention efficiencies. (A) Sample recovered from inside the crater

626 following a $45^{\circ}$ experiment; numbers correspond to microprobe analyses in Table 6. (B) Sample

627 recovered beyond the crater rim following a $90^{\circ}$ experiment. In both (A) and (B) the uniform

628 backscatter brightness indicates little to no chemical variation in the glasses. 


\section{Notes on Desired Figure Sizes}

631

632

633

634

635

636

637

638

639

Figure 1 = two columns wide

Figure 2 = two columns wide

Figure $3=$ one column wide

Figure $4=$ one column wide

Figure $5=$ one column wide

Figure $6=$ two columns wide

Figure $7=$ one column wide

Figure $8=$ two columns wide

Figure $9=$ one column wide 
Table 1. Summary of experiments.

\begin{tabular}{llll}
\hline Projectile & Target & Impact Angle $\left(^{\circ}\right)$ & Impact Speed $\left(\mathrm{km} \mathrm{s}^{-1}\right)$ \\
\hline Basalt & Pumice & 90 & 4.47 \\
& & 60 & 4.93 \\
& 45 & 4.44 \\
Aluminum & Pumice & 90 & 4.53 \\
\hline
\end{tabular}

640

Table 2. End member compositions for chemical mixing models (wt. \%)

\begin{tabular}{cccccccccccc}
\hline End member & $\mathrm{Na}_{2} \mathrm{O}$ & $\mathrm{MgO}$ & $\mathrm{SiO}_{2}$ & $\mathrm{Al}_{2} \mathrm{O}_{3}$ & $\mathrm{P}_{2} \mathrm{O}_{5}$ & $\mathrm{~K}_{2} \mathrm{O}$ & $\mathrm{CaO}$ & $\mathrm{TiO}_{2}$ & $\mathrm{MnO}$ & $\mathrm{FeO}$ & Total \\
\hline Pumice Target & 4.07 & 0.10 & 76.20 & 12.32 & 0.01 & 4.75 & 0.61 & 0.05 & 0.04 & 1.09 & 99.24 \\
Basalt Projectile & 3.55 & 3.95 & 53.10 & 14.41 & 1.34 & 1.90 & 8.09 & 2.64 & 0.22 & 13.05 & 102.25
\end{tabular}

Table 3. Bulk compositions of projectile-contaminated pieces (wt. \%).

\begin{tabular}{|c|c|c|c|c|c|c|c|c|c|c|c|c|}
\hline Angle $\left(^{\circ}\right)$ & Location & $\mathrm{Na}_{2} \mathrm{O}$ & $\mathrm{MgO}$ & $\mathrm{SiO}_{2}$ & $\mathrm{Al}_{2} \mathrm{O}_{3}$ & $\mathrm{P}_{2} \mathrm{O}_{5}$ & $\mathrm{~K}_{2} \mathrm{O}$ & $\mathrm{CaO}$ & $\mathrm{TiO}_{2}$ & $\mathrm{MnO}$ & $\mathrm{FeO}$ & Total \\
\hline 90 & Inside crater $^{\mathrm{a}}$ & 4.10 & 1.15 & 74.73 & 13.39 & 0.27 & 4.20 & 2.18 & 0.58 & 0.08 & 3.72 & 104.41 \\
\hline 60 & Beyo & 3.8 & 0.9 & 71.45 & 12. & 0.22 & 4.13 & 1. & 0.46 & 8 & 3. & 98.82 \\
\hline 60 & Inside crater & 3.85 & 0.32 & 72.96 & 12.15 & 0.04 & 4.39 & 0.87 & 0.14 & 0.05 & 1.58 & 96.35 \\
\hline 45 & Beyond rim ${ }^{b}$ & 3.90 & 0.66 & 73.38 & 12.38 & 0.06 & 4.46 & 0.99 & 0.18 & 0.06 & 1.92 & 97.97 \\
\hline 30 & Beyond rim ${ }^{\mathrm{b}}$ & 4.00 & 0.36 & 75.95 & 12.78 & 0.05 & 4.60 & 0.95 & 0.17 & 0.05 & 1.68 & 100.60 \\
\hline
\end{tabular}

$642{ }^{a}$ This experiment produced insufficient projectile-contaminated pieces located beyond the crater rim to do both ICP-AES and microprobe analyses 643 on that material.

$644{ }^{\mathrm{b}}$ This experiment produced insufficient projectile-contaminated pieces located inside the crater to do both ICP-AES and microprobe analyses on 645 that material.

Table 4. Amount of projectile retained in and near crater

\begin{tabular}{|c|c|c|c|c|}
\hline Angle $\left(^{\circ}\right)$ & Location & $\begin{array}{l}\text { Mass Fraction of } \\
\text { Projectile Retained }\end{array}$ & $X_{\text {Bas }}$ & $r^{2}$ \\
\hline 90 & Inside crater & $0.72 \pm 0.01$ & $0.21 \pm 0.002$ & 0.9998 \\
\hline 60 & Beyond rim & $0.23 \pm 0.02$ & $0.19 \pm 0.008$ & 0.9971 \\
\hline 60 & Inside crater & $0.05 \pm 0.03$ & $0.04 \pm 0.003$ & 0.9912 \\
\hline 45 & Beyond rim & $0.14 \pm 0.04$ & $0.07 \pm 0.006$ & 0.9865 \\
\hline 30 & Beyond rim & $0.07 \pm 0.02$ & $0.05 \pm 0.002$ & 0.9966 \\
\hline
\end{tabular}


Table 5. Compositions from electron microprobe analyses (wt. \%).

\begin{tabular}{|c|c|c|c|c|c|c|c|c|c|c|c|c|c|}
\hline Location & $\mathrm{Na}_{2} \mathrm{O}$ & $\mathrm{MgO}$ & $\mathrm{SiO}_{2}$ & $\mathrm{Al}_{2} \mathrm{O}_{3}$ & $\mathrm{P}_{2} \mathrm{O}_{5}$ & $\mathrm{~K}_{2} \mathrm{O}$ & $\mathrm{CaO}$ & $\mathrm{TiO}_{2}$ & $\mathrm{MnO}$ & $\mathrm{FeO}$ & $\mathrm{CuO}$ & $\mathrm{ZnO}$ & Total \\
\hline Fig. 7A (1) & 3.74 & 0.01 & 76.85 & 12.54 & 0.02 & 4.86 & 0.52 & 0.07 & 0.00 & 0.84 & 0.00 & 0.02 & 99.46 \\
\hline Fig. 7B $(1)^{*}$ & 0.00 & 0.01 & 30.75 & 66.09 & 0.04 & 0.01 & 0.00 & 0.03 & 0.04 & 0.00 & 1.99 & 0.04 & 99.00 \\
\hline Fig. 7B (2) & 4.46 & 0.13 & 69.57 & 17.24 & 0.03 & 6.73 & 0.57 & 0.06 & 0.06 & 1.04 & 0.00 & 0.00 & 99.87 \\
\hline Fig. 7B (3) & 5.29 & 0.05 & 69.91 & 16.03 & 0.03 & 5.09 & 1.12 & 0.03 & 0.06 & 0.87 & 0.08 & 0.00 & 98.56 \\
\hline Fig. 7C (1) & 4.05 & 0.04 & 76.18 & 12.41 & 0.02 & 5.09 & 0.61 & 0.05 & 0.00 & 1.15 & 0.00 & 0.00 & 99.61 \\
\hline Fig. 7C (2)* & 0.00 & 0.00 & 15.52 & 81.08 & 0.05 & 0.00 & 0.01 & 0.04 & 0.12 & 0.03 & 1.76 & 0.00 & 98.62 \\
\hline Fig. 8B (1) & 3.88 & 0.02 & 77.83 & 12.87 & 0.00 & 4.91 & 0.46 & 0.07 & 0.00 & 0.88 & $\mathrm{~N} / \mathrm{A}$ & N/A & 100.93 \\
\hline Fig. 8B (2) & 3.95 & 0.03 & 77.81 & 12.88 & 0.00 & 4.72 & 0.55 & 0.06 & 0.00 & 0.92 & $\mathrm{~N} / \mathrm{A}$ & $\mathrm{N} / \mathrm{A}$ & 100.92 \\
\hline Fig. 8B (3) & 1.33 & 7.44 & 43.56 & 5.05 & 1.41 & 0.71 & 11.53 & 5.66 & 0.39 & 22.49 & $\mathrm{~N} / \mathrm{A}$ & N/A & 99.64 \\
\hline Fig. 8B (4) & 5.88 & 0.14 & 56.78 & 26.46 & 0.01 & 0.71 & 9.76 & 0.15 & 0.00 & 0.96 & $\mathrm{~N} / \mathrm{A}$ & N/A & 100.84 \\
\hline Fig. 8D (1) & 3.50 & 0.03 & 76.85 & 12.93 & 0.00 & 4.56 & 0.55 & 0.05 & 0.04 & 0.92 & $\mathrm{~N} / \mathrm{A}$ & $N / A$ & 99.47 \\
\hline Fig. 8D (2) & 1.65 & 11.28 & 47.02 & 5.59 & 1.17 & 0.70 & 7.72 & 1.90 & 0.45 & 20.97 & $\mathrm{~N} / \mathrm{A}$ & $N / A$ & 98.45 \\
\hline Fig. 8F (1) & 1.61 & 8.35 & 42.26 & 7.01 & 0.79 & 0.69 & 5.49 & 3.94 & 0.46 & 28.21 & $\mathrm{~N} / \mathrm{A}$ & $N / A$ & 98.81 \\
\hline Fig. 8F (2) & 3.90 & 0.03 & 76.29 & 12.81 & 0.01 & 4.65 & 0.56 & 0.06 & 0.09 & 1.10 & $\mathrm{~N} / \mathrm{A}$ & $\mathrm{N} / \mathrm{A}$ & 99.50 \\
\hline Fig. 8F (3) & 0.01 & 21.99 & 34.54 & 0.02 & 0.16 & 0.01 & 0.32 & 0.10 & 0.91 & 42.30 & $\mathrm{~N} / \mathrm{A}$ & $N / A$ & 100.41 \\
\hline Fig. $8 \mathrm{~F}(4)$ & 3.86 & 0.03 & 76.26 & 12.79 & 0.01 & 4.66 & 0.56 & 0.08 & 0.09 & 1.06 & $\mathrm{~N} / \mathrm{A}$ & $N / A$ & 99.38 \\
\hline
\end{tabular}

*These compositions are reported on a metals basis because they are metallic regions in the analyzed projectile-contaminated piece.

Table 6. Compositions from electron microprobe analyses (wt. \%) and mixing model results.

\begin{tabular}{|c|c|c|c|c|c|c|c|c|c|c|c|c|c|}
\hline Location & $\mathrm{Na}_{2} \mathrm{O}$ & $\mathrm{MgO}$ & $\mathrm{SiO}_{2}$ & $\mathrm{Al}_{2} \mathrm{O}_{3}$ & $\mathrm{P}_{2} \mathrm{O}_{5}$ & $\mathrm{~K}_{2} \mathrm{O}$ & $\mathrm{CaO}$ & $\mathrm{TiO}_{2}$ & $\mathrm{MnO}$ & $\mathrm{FeO}$ & Total & $r^{2}$ & $P$ value* \\
\hline Fig. 10 (1) & 3.60 & 0.03 & 77.28 & 12.87 & 0.02 & 4.70 & 0.54 & 0.06 & 0.06 & 0.91 & 100.11 & 0.0252 & 0.64 \\
\hline Fig. 10 (2) & 3.50 & 0.02 & 77.30 & 12.89 & 0.01 & 4.70 & 0.48 & 0.05 & 0.06 & 0.95 & 100.00 & 0.0299 & 0.61 \\
\hline Fig. 10 (3) & 3.93 & 0.12 & 76.21 & 12.69 & 0.04 & 4.49 & 1.30 & 0.06 & 0.06 & 2.56 & 101.54 & 0.0138 & 0.73 \\
\hline Fig. 10 (4) & 3.49 & 2.13 & 71.97 & 11.95 & 0.03 & 3.63 & 3.20 & 0.08 & 0.06 & 3.39 & 100.11 & 0.0987 & 0.35 \\
\hline Fig. 10 (5) & 3.93 & 0.03 & 76.86 & 12.85 & 0.01 & 4.65 & 0.52 & 0.07 & 0.04 & 1.01 & 99.97 & 0.0275 & 0.63 \\
\hline
\end{tabular}

${ }^{*} P$ value calculated from a $t$-test about the significance of the slope of the regression line $\left(X_{B a s}\right)$. All values are $>0.05$, indicating

that any linear relationship is statistically insignificant. There is no robust evidence for two-component mixing in these analyses. 